\title{
Water Protects Graphitic Surface from Airborne
}

\section{Hydrocarbon Contamination}

Zhiting Li ${ }^{a \ddagger}$, Andrew Kozbial ${ }^{b \ddagger}$, Nikoloz Nioradze ${ }^{\mathrm{a}}$, David Parobek ${ }^{\mathrm{a}}$, Ganesh Jagadeesh Shenoy ${ }^{\mathrm{a}}$, Muhammad Salim ${ }^{\mathrm{a}}$, Shigeru Amemiya ${ }^{\mathrm{a}}, \mathrm{Lei}_{\mathrm{Li}}^{\mathrm{b}, \mathrm{c}^{*}}$ and Haitao Liu ${ }^{\mathrm{a}^{*}}$

${ }^{a}$ Department of Chemistry, University of Pittsburgh, Pittsburgh, PA 15260, USA

${ }^{\mathrm{b}}$ Department of Chemical \& Petroleum Engineering, Swanson School of Engineering, University of Pittsburgh, Pittsburgh, PA 15261, USA

${ }^{\mathrm{c}}$ Department of Mechanical Engineering \& Materials Science, Swanson School of Engineering, University of Pittsburgh, Pittsburgh, PA 15261, USA

‡: These authors contribute equally to this work

*: Corresponding author. Email: hliu@pitt.edu (H. L.); lel55@pitt.edu (L.L.)

\section{Supporting Information}

\section{Spectroscopic ellipsometry (SE) analysis}

Ellipsometry measurements were applied to monitor the in-situ film growth on freshly exfoliated HOPG surface. In this technique, two parameters, $\Psi$ and $\Delta$, were measured which reflects the polarization state change of light upon reflection from a sample. For example, $\Delta$ is related to the phase difference between parallel (p) and perpendicular (s) 
polarized light and $\Psi$ to the amplitude ratio of p- and s-polarized components. For an exfoliated HOPG surface exposed in ambient air at room temperature (r.t.), measured values of $\Psi$ and $\Delta$ are shown in Supplementary Fig. 1 ranging from 380-900 nm wavelength. Both $\Psi$ and $\Delta$ showed a monotonic increase at all wavelengths as a function of air exposure time, indicating an adsorption process onto the fresh HOPG surface. ATR-FTIR (see main text) demonstrates that the adsorbed molecules are hydrocarbons. In particular, $\Delta$ is more sensitive to surface changes especially at lower wavelengths, consistent with previous studies on alkylsilyl (sub)monolayers. ${ }^{\mathrm{S} 1}$, S2 In comparison, $\Psi$ and $\Delta$ showed almost negligible change after 5 minute low temperature storage as shown in Supplementary Fig. 2, indicating a sub-monolayer water film on HOPG surface. Due to the existence of such water film, it took about 4 hours to reach the same $\Psi$ and $\Delta$ values compared to only 60 minutes on HOPG without low temperature treatment.

Given that the ellipsometry measured $\Psi$ and $\Delta$ values only reflect the optical properties of a surface, an optical model is therefore necessary to translate $\Psi$ and $\Delta$ into physical meaning (e.g., film thickness). The detailed model analysis of a HOPG surface with/without hydrocarbon adsorption can be found in our previous work. ${ }^{\mathrm{S} 3}$ In brief, a layer-by-layer structure of adsorbed hydrocarbon films on HOPG was assumed during ambient exposure at r.t., where hydrocarbon layer and HOPG were modeled using a bspline and Cauchy model, respectively. Similarly, a sandwich structure of hydrocarbon/water/HOPG was considered after low temperature storage while water model was applied. The schematic images of both models are shown in Supplementary Fig. 3. 
Further explanations about the layer-by-layer deposition model (Fig. 5 and Supplementary Fig. 3) are as follows: during the first 10 minutes of low temperature treatment, we simply attribute all the thickness increase to the formation of water film. This can be explained by the fact that HOPG surface showed a constantly low hydrocarbon adsorption rate $(0.002 \mathrm{~nm} /$ minute $)$ at low temperature. Thus an estimated hydrocarbon thickness of $c a .0 .02 \mathrm{~nm}$ is expected during the first 10 minutes of low temperature treatment, which only contributes $25 \%$ to the observed $0.08 \mathrm{~nm}$ thickness. In addition, we found that HOPG stored at low temperature over different time (10-30 minutes) resulted in a consistent thickness increase of $0.06-0.10 \mathrm{~nm}$ (vide infra), indicative of submonolayer to monolayer water film on HOPG surface as confirmed by many other studies. ${ }^{54-8}$ In this regard, we believe that the observed thickness increase during the low temperature treatment is dominated by the condensation of water layer. In fact, we also tentatively alternated the model from HOPG/water to HOPG/hydrocarbon and only found a slight increase by $10 \%$ in modified thickness, further validating the approximation that we proposed in the ellipsometry model.

\section{Cyclic Voltammetry (CV) on HOPG surface}

HOPG was of SPI-1 $(10 \times 10 \times 1 \mathrm{~mm})$ grade, obtained from SPI Supplies (West Chester, PA). Fresh surface of HOPG was exposed by scotch tape peeling. Homogenous coverage of scotch tape by HOPG flakes was inspected under microscope to insure that the whole HOPG surface was exfoliated. 
An electrochemical cell was assembled by sandwiching HOPG between two Teflon plates exposing $6 \mathrm{~mm}$ disk to the solution and attaching the glass wall to the o-ring placed in the top Teflon plate. The cell was covered by a Teflon cap to minimize the contamination of the aqueous electrolyte solution with airborne organic impurities. Prior to use, the Teflon-glass electrochemical cell was cleaned in piranha solution (a 1:3 mixture of $30 \% \mathrm{H}_{2} \mathrm{O}_{2}$ and $95.0-98.0 \% \mathrm{H}_{2} \mathrm{SO}_{4}$ ), then washed in Milli-Q water and dried in air (Caution: piranha solution reacts violently with organics and should be handled with extreme care!). Pt wire (99.9\%, Alfa Aesar) was used as a counter electrode and $\mathrm{Ag} / \mathrm{AgCl}$ wire as a reference electrode.

Aqueous solution prepared using high purity water (Milli-Q, TOC $=3 \mathrm{ppb}$ ) with a resistivity of $18.2 \mathrm{M} \Omega \mathrm{cm}$ at $25^{\circ} \mathrm{C}$, contained $1.0 \mathrm{mM}$ potassium ferrocyanide trihydrate $\left(\mathrm{K}_{4} \mathrm{Fe}(\mathrm{CN})_{6} \cdot 3 \mathrm{H}_{2} \mathrm{O} ; 99 \%\right.$, Fisher Scientific) and $1.0 \mathrm{M}$ potassium chloride $(\mathrm{KCl}$; SigmaAldrich, $>99 \%$ ) as supporting electrolyte, the scan rate for CVs was $0.1 \mathrm{~V} / \mathrm{s}$.

\section{XPS C1s peak analysis}

To estimate the contribution of the top layer of HOPG, we make the following assumptions:

1. While the x-ray penetrates into the sample, there is a limited escape depth of the ejected electrons. Based on the energy of our x-ray gun ( $c a .1400 \mathrm{eV})$, the depth is around $10 \mathrm{~nm},{ }^{\mathrm{S} 9}$ corresponding to $c a .29$ graphene layers in total.

2. According to Beer-Lambert relationship: $I_{o}=I / \exp \left(-\left(d_{\mathrm{G}} / \cos q\right) / \lambda\right)$, where $I_{o}(I)$ attributes to the peak intensity of top (second) graphene layer, $d_{G}=3.5 \AA$ is 
the thickness of graphene layer and $q$ is the escape angle $\left(45^{\circ}\right), \lambda$ is the Inelastic Mean Free Path (IMFP) and equals $24.8 \AA .{ }^{\text {S10 }}$

With these assumptions, the contribution of the $\mathrm{k}^{\text {th }}(\mathrm{k}<30)$ layer of graphene to the observed C1s peak will decrease to $0.819^{\mathrm{k}-1}$ for each increase of layer depth. As a result, the contribution from the top layer of HOPG accounts for $18 \%$ of the observed C1s peak.

\section{Estimation of hydrocarbon coverage}

According to our WCA data (Fig 1), the hydrocarbon adsorption becomes saturated on HOPG surface with an increase of WCA by $c a .30^{\circ}$. In comparison, HOPG stored at low temperature $\left(-15^{\circ} \mathrm{C}\right)$ only shows a slight increase of WCA by $c a \cdot 10^{\circ}$. Assuming that the WCA increase is in linear correlation with hydrocarbon adsorption kinetics, we estimate the hydrocarbon coverage to be $c a$. $30 \%$ on HOPG stored at low temperature for 2 hours.
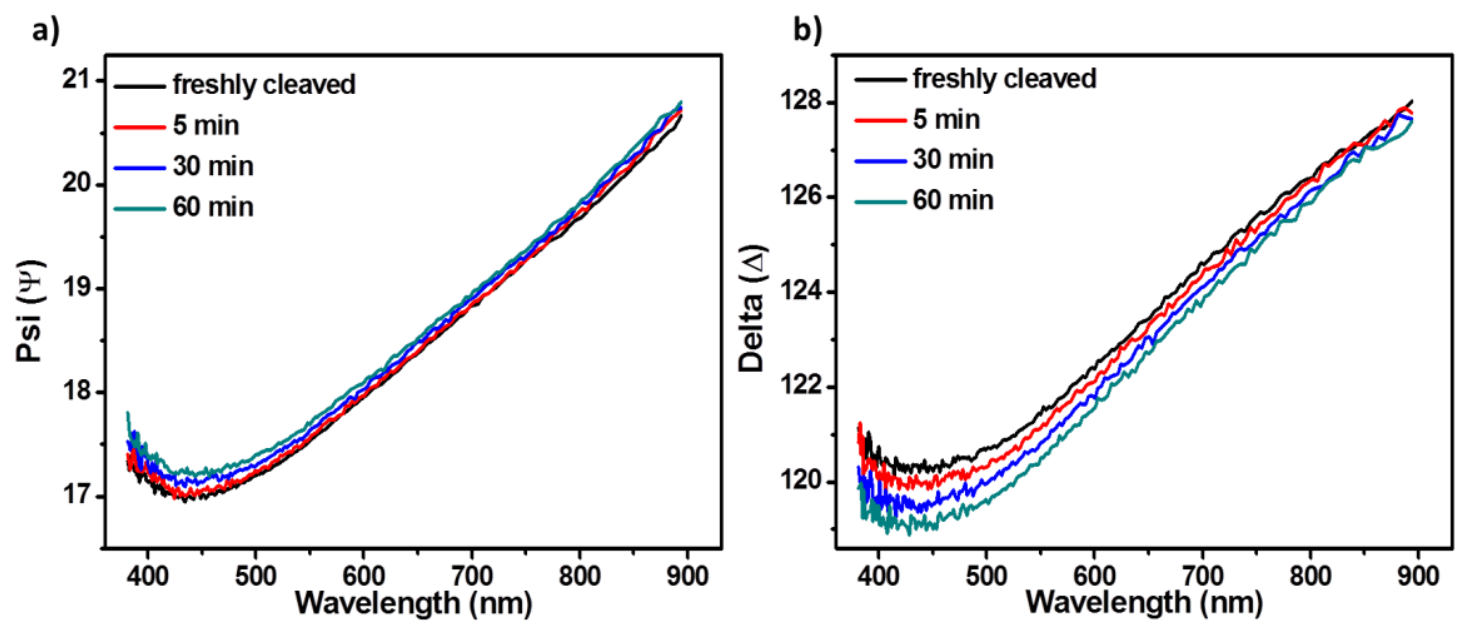

Supplementary Figure 1. Temporal monitoring of ellipsometric quantities (a) Psi ( $\Psi$ ) and (b) Delta $(\Delta)$ after HOPG exfoliation. 
a)

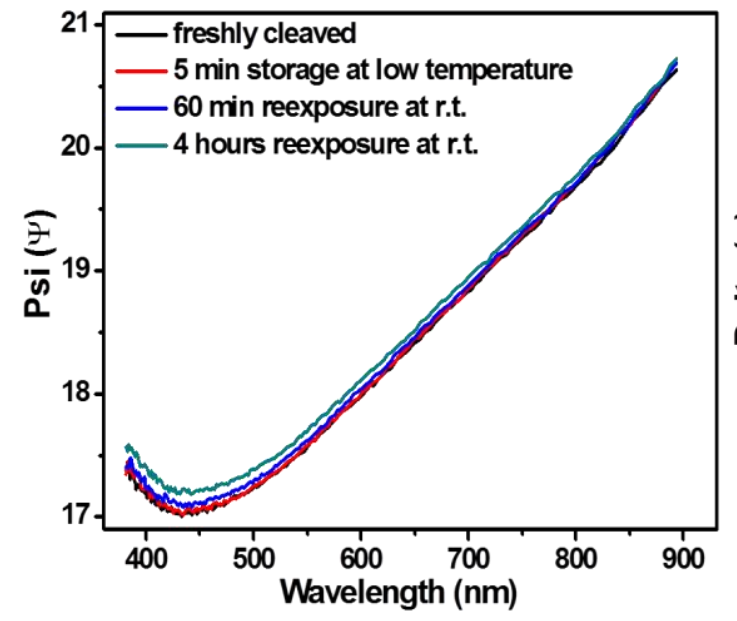

b)

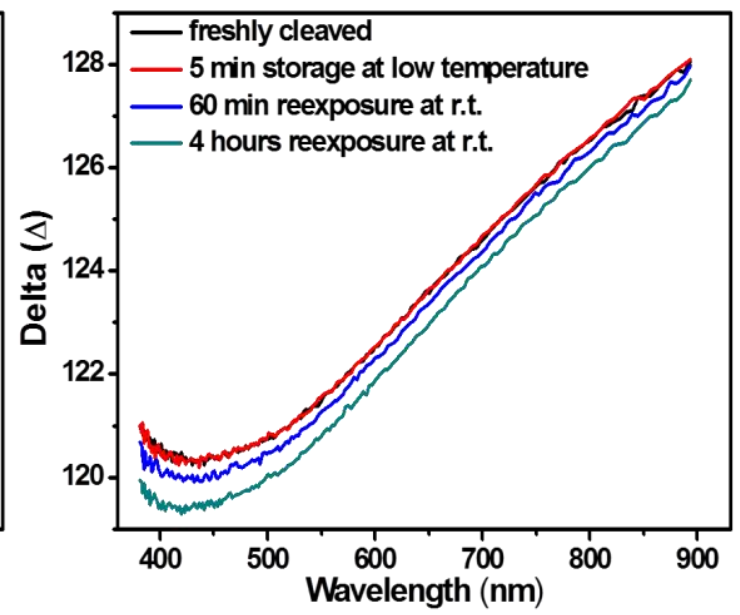

Supplementary Figure 2. Temporal monitoring of ellipsometric quantities (a) Psi ( $\Psi$ ) and (b) Delta $(\Delta)$ of exfoliated HOPG before and after 5 minute low temperature storage.

a)

Ambient air

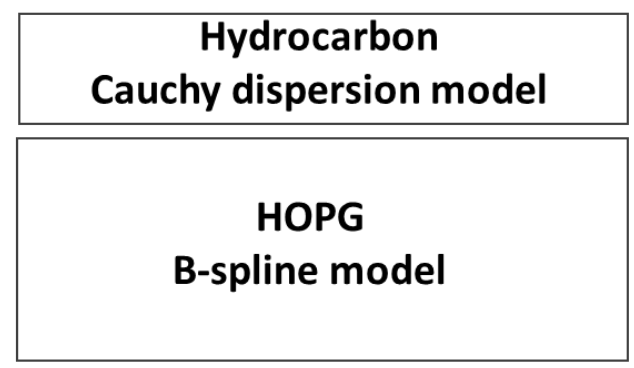

b)

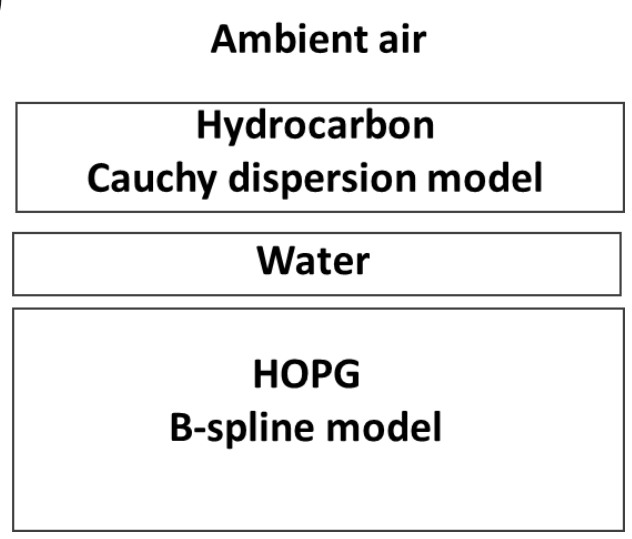

Supplementary Figure 3. The schematic model used for ellipsometric measurement of (a) hydrocarbon adsorbed on freshly exfoliated HOPG surface and (b) hydrocarbon adsorbed on low temperature treated HOPG surface. 


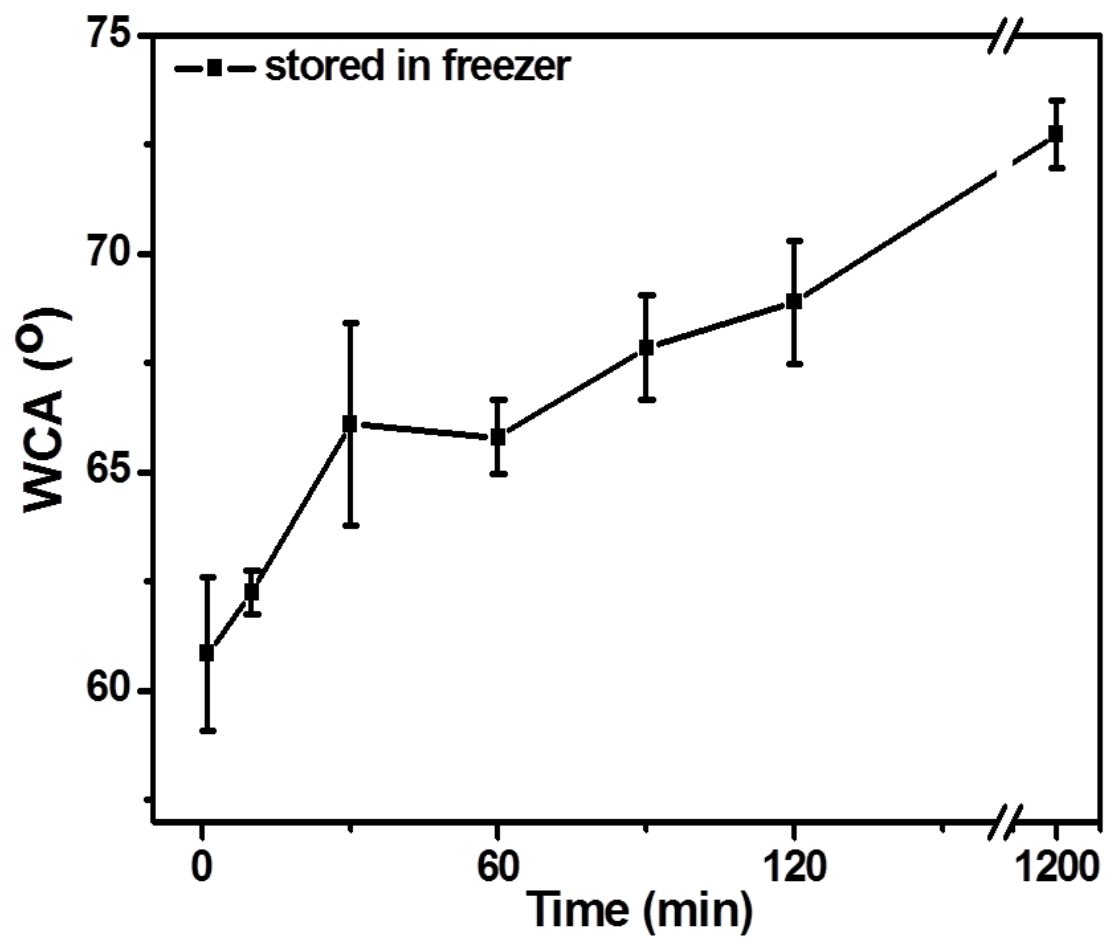

Supplementary Figure 4. Temporal evolution of the WCA measured on freshly exfoliated $\mathrm{HOPG}$ surface stored in freezer. The local temperature was measured to be $-15^{\circ} \mathrm{C}$.
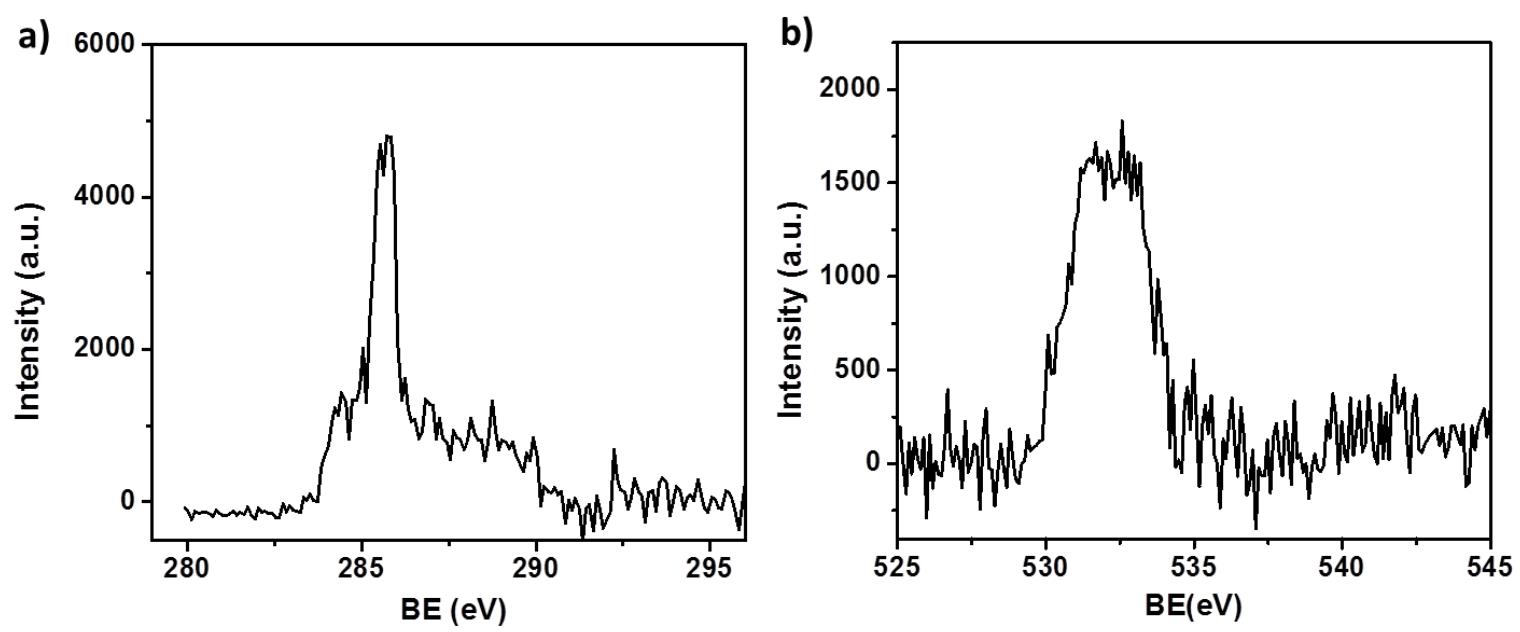

Supplementary Figure 5. (a) Carbon 1s and (b) Oxygen 1s peak difference between two HOPG samples stored at room temperature and low temperature for 6 days. 

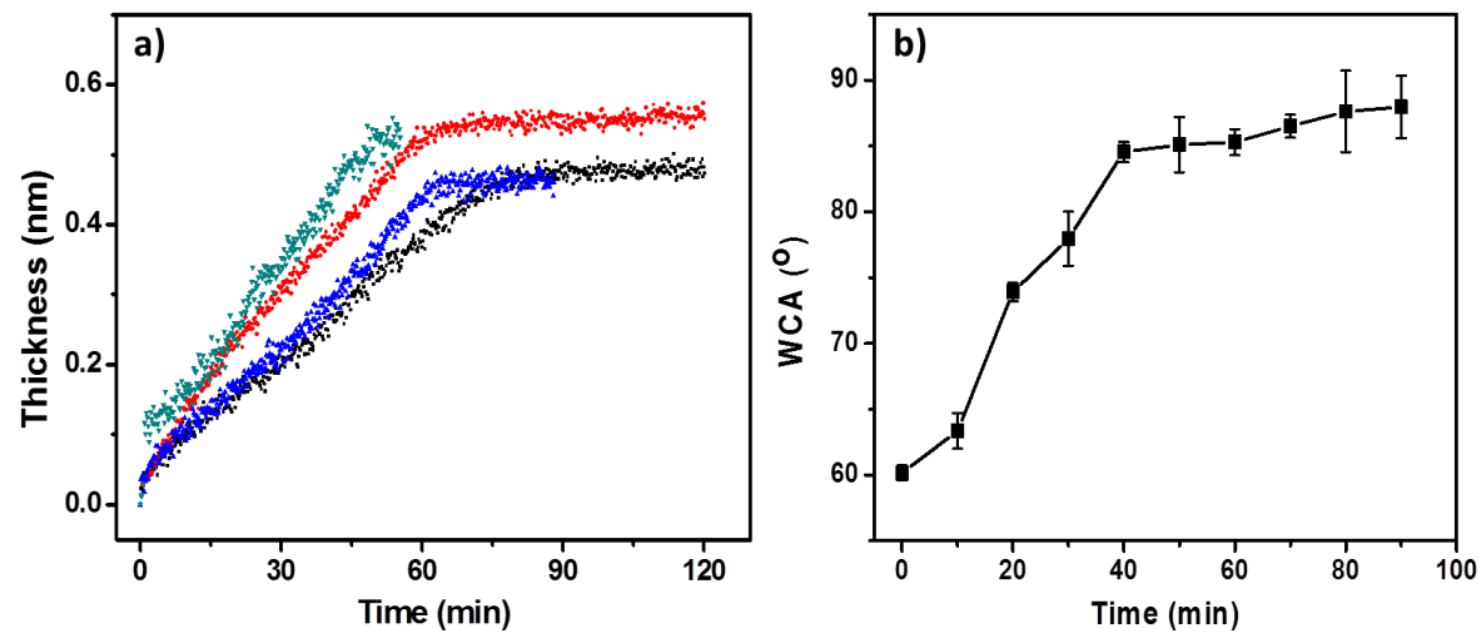

Supplementary Figure 6. (a) Ellipsometry and (b) WCA measurements on freshly exfoliated HOPG surface exposed in air at room temperature in the same laboratory.
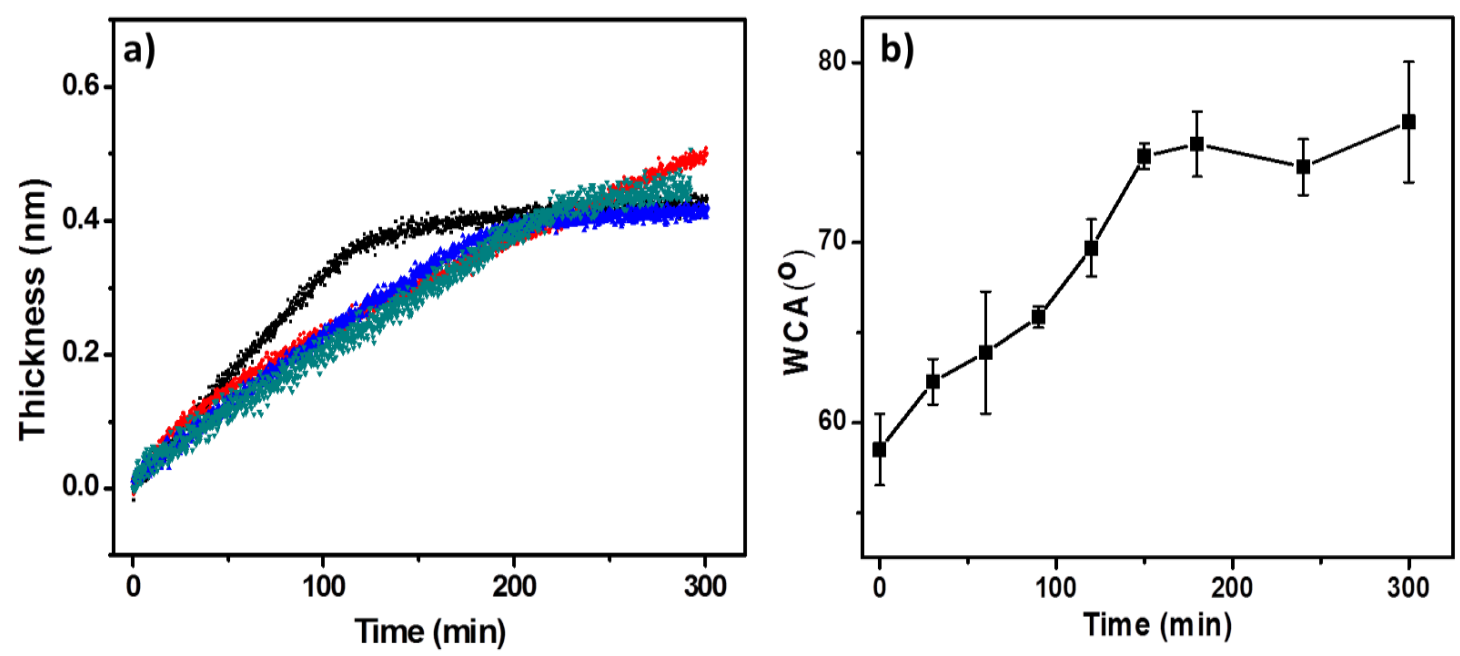

Supplementary Figure 7. (a) Ellipsometry and (b) WCA measurements on freshly exfoliated HOPG surface exposed in air at low temperature in the same laboratory. 
Opt. Const. of Cauchy vs. nm

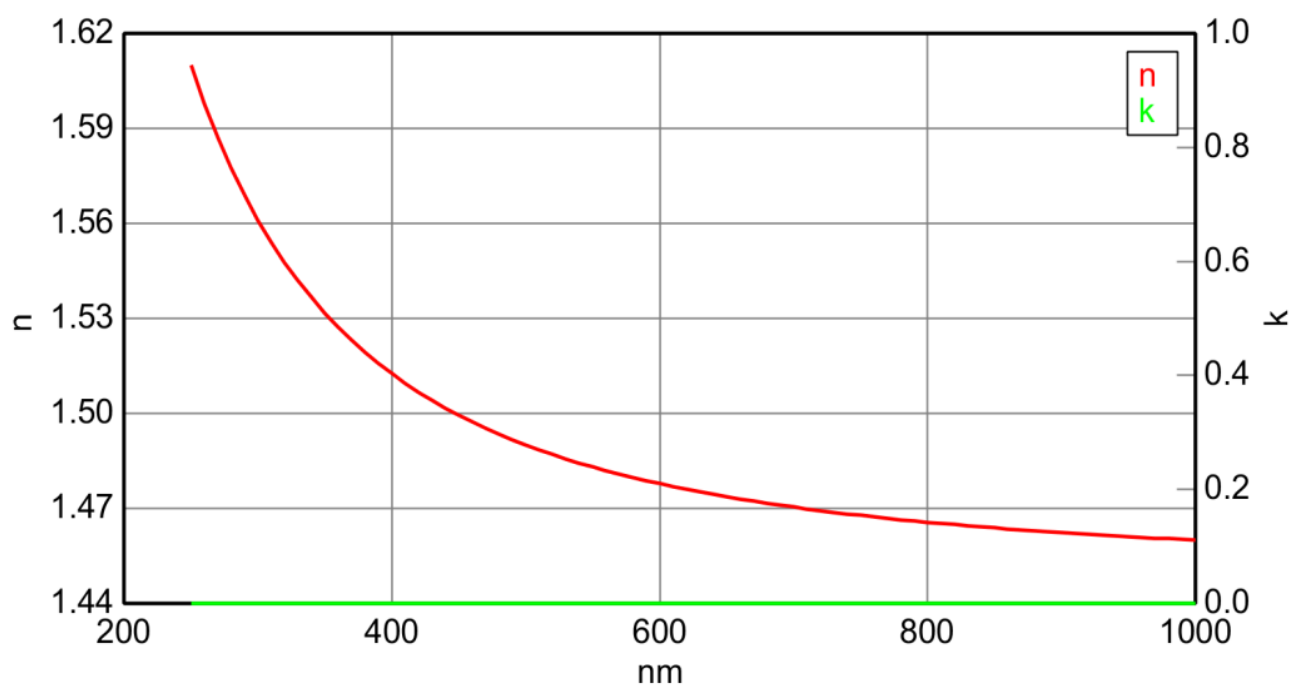

Opt. Const. of $\mathrm{H} 2 \mathrm{O}$ vs. $\mathrm{nm}$

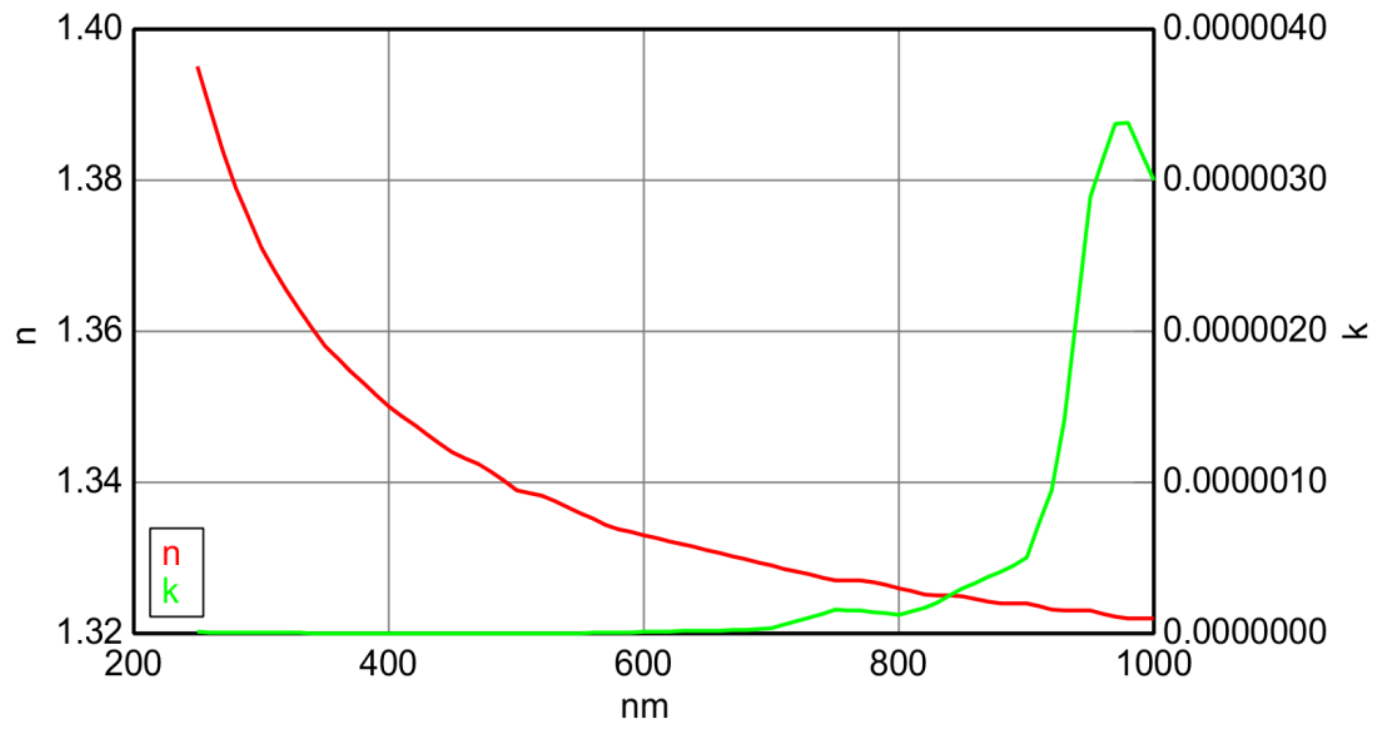

Supplementary Figure 8. Index of refraction (n) and extinction coefficient (k) for both the Cauchy model and water model. Ellipsometry measurements (Supplementary Fig. 2) were collected at wavelength of 380 to $900 \mathrm{~nm}$. Index of refraction (n) is slightly higher (ca. 10\%) in Cauchy model compared to water model; whereas, extinction coefficient $(\mathrm{k})$ of Cauchy model is defined as 0 for all wavelengths and very near or slightly above 0 for water model, indicating a transparent layer. Figure and optical constants taken from CompleteEASE supplied by J.A. Woollam Co. 


\section{Supplementary References}

S1. Nabok, A.; Tsargorodskaya, A. The Method of Total Internal Reflection Ellipsometry for Thin Film Characterisation and Sensing. Thin Solid Films 2008, 516, 8993-9001.

S2. $\quad$ Kriechbaumer, V.; Tsargorodskaya, A.; Mustafa, Mohd K.; Vinogradova, T.; Lacey, J.; Smith, David P.; Abell, Benjamin M.; Nabok, A. Study of ReceptorChaperone Interactions Using the Optical Technique of Spectroscopic Ellipsometry. Biophys. J. 2011, 101, 504-511.

S3. $\quad$ Kozbial, A.; Li, Z.; Sun, J.; Gong, X.; Zhou, F.; Wang, Y.; Xu, H.; Liu, H.; Li, L. Understanding the Intrinsic Water Wettability of Graphite. Carbon 2014, 74, 218-225.

S4. Chakarov, D. V.; Österlund, L.; Kasemo, B. Water Adsorption on Graphite (0001). Vacuum 1995, 46, 1109-1112.

S5. $\quad$ Cao, P.; Xu, K.; Varghese, J. O.; Heath, J. R. The Microscopic Structure of Adsorbed Water on Hydrophobic Surfaces under Ambient Conditions. Nano Lett. 2011, 11, 5581-5586.

S6. Cabrera Sanfelix, P.; Holloway, S.; Kolasinski, K. W.; Darling, G. R. The Structure of Water on the (0001) Surface of Graphite. Surf. Sci. 2003, 532$535,166-172$.

S7. Teschke, O. Imaging Ice-Like Structures Formed on HOPG at Room Temperature. Langmuir 2010, 26, 16986-16990.

S8. Morimoto, T.; Miura, K. Adsorption Sites for Water on Graphite. 1. Effect of High-Temperature Treatment of Sample. Langmuir 1985, 1, 658-662.

S9. Ji, X.; Banks, C. E.; Xi, W.; Wilkins, S. J.; Compton, R. G. Edge Plane Sites on Highly Ordered Pyrolytic Graphite as Templates for Making Palladium Nanowires Via Electrochemical Decoration. J. Phys. Chem. B 2006, 110, 22306-22309.

S10. Lai, S. C. S.; Patel, A. N.; McKelvey, K.; Unwin, P. R. Definitive Evidence for Fast Electron Transfer at Pristine Basal Plane Graphite from High-Resolution Electrochemical Imaging. Angew. Chem. Int. Ed. 2012, 51, 5405-5408. 